\title{
1. Combinatorial Exploration of the High 2 Entropy Alloy System Co-Cr-Fe-Mn-Ni
}

Alexander Kauffmann ${ }^{1}$, Michael Stüber ${ }^{2}$, Harald Leiste ${ }^{2}$, Sven Ulrich ${ }^{2}$, Sabine Schlabach1,3, Dorothée Vinga Szabó1,3, Sascha Seils¹, Bronislava Gorr ${ }^{4}$, Hans Chen ${ }^{1}$, Hans-Jürgen Seifert ${ }^{2}$, Martin Heilmaier ${ }^{1}$

${ }^{1}$ Karlsruhe Institute of Technology (KIT), Institute for Applied Materials (IAM-WK), Germany

${ }^{2}$ Karlsruhe Institute of Technology (KIT), Institute for Applied Materials (IAM-AWP), Germany

${ }^{3}$ Karlsruhe Institute of Technology (KIT), Karlsruhe Nano Micro Facility (KNMF), Germany

${ }^{4}$ University of Siegen, Germany

\section{Abstract}

In high entropy alloys, the number of base alloying elements is increased to at least five and their individual concentrations are rather high in comparison to conventional metallic alloys. This strategy aims at maximization of the configurational part of entropy and stabilization of disordered, singlephase solid solutions with simple crystal structure. In the present contribution, a first attempt is presented for the exploration of the phase field of the face centered cubic solid solution in the vicinity of the well-known equimolar composition of $\mathrm{CoCrFeMnNi}$ (Cantor alloy) on the basis of combinatorial thin film deposition from sectioned, circular targets by magnetron sputtering. A variation of the chemical composition of the thin films from almost binary systems for substrates (placed on circular positions at the rim of the coated area) towards thin films with an almost equimolar composition (i.e. obtained for samples coated at the center region of the target) was achieved. Crystal structures of the binary thin films were studied and the according lattice parameters of body centered cubic, face centered cubic, hexagonally closed packed and complex cubic ( $\alpha$-Mn prototype) crystal structures are in good accordance with expectations for solid solutions from literature data. The microstructure of the face centered solid solution thin films deposited at the center region of the target was investigated in detail by transmission electron microscopy and atom probe tomography. An ultra- 
fine grained, columnar microstructure was found exhibiting disorder down to atomic length scale. Thus, a suitable platform for future investigations of the entire face centered cubic phase field is provided and the strength of the combinatorial thin film approach for the investigation of complex microstructure development in high entropy alloy systems is revealed.

\section{Introduction}

The concept of high entropy alloys (HEA) differs from conventional alloy design strategies by increasing the number of base elements as well as their concentrations. Rather than being composed of a single principal element and minor additions for obtaining desired microstructures and materials properties, this new class of materials is chemically complex. It is composed of multiple principal elements, usually in almost equiatomic ratios [1,2]. Here, the basic intention is to increase the configurational part of entropy $\Delta S_{\text {config }}=-k_{B} \cdot \sum_{i=1}^{n} \ln x_{i}$ (with $k_{B}$ as Boltzmann constant, $x_{i}$ as the molar concentration of species $i$ and $n$ as the number of elements) towards its maximum value of $\Delta S_{\text {config }}^{\max }=k_{B} \cdot \ln n$ (with equimolar composition $x_{i}=1 / n$ ). This procedure should stabilize disordered, single-phase materials with simple crystal structure such as face-centered cubic (FCC), body-centered cubic (BCC) and hexagonally closed packed (HCP). Examples found so far which follow this assumption are CoCrFeMnNi (FCC) [1], AuCuNiPdPt (FCC) [3], MoNbTaVW (BCC) [4], HfNbTaTiZr (BCC) [5] and DyGdHoTbY (HCP) [6]. Accordingly, ordered intermetallic phases are suppressed in this kind of alloys. Nevertheless, the configurational entropy is not the sole decisive factor for the thermodynamic stability of particular phases, as the vast majority of multi-phase compositionally complex alloys reveals - most alloys do not exclusively exhibit simple solid solutions [7-13]. Motivated by fundamental investigations for future materials development, compositions different from equimolar concentrations are of central interest, for example for the development of high temperature materials or wear resistant alloys. This might for example be driven by controlled precipitation of intermetallic phases from supersaturated solid solution [14-16] or by small additions of supplemental elements for subtle property adjustment $[17,18]$ as well as by studying compositiondepending effects like stacking fault energy or solid solution hardening [19]. Hence, efficient theoretical and experimental methods have to be conducted in order to screen the appearance of phase 
fields in the center of five or more component systems. Beside from diffusion multiples as a common tool which are already employed in the case of HEAs [20], combinatorial thin film deposition techniques provide innovative high-throughput platforms which fit these requirements at best [21]. Here, a first substantial study of HEAs was performed by Marshal et al. [22] revealing that the BCC solid solution in $\mathrm{AlCoCrFeMn}$ is formed for an $\mathrm{Al}$ content of \pm 6 at $\%$ around the equiatomic composition. In contrast to the aforementioned diffusion multiples, thin film deposition methods provide the possibility to explore and investigate samples prepared under highly non-equilibrium growth conditions. Hence, variable film deposition parameters as well as further heat-treatments of asdeposited thin films offer additional degrees of freedom.

In the present contribution we are focusing on $\mathrm{CoCrFeMnNi}$ - the so-called Cantor alloy, which crystallizes with FCC structure [1]. It is one of the few examples of HEAs which can be tailored by means of classical metallurgical processes [23-25]. Thus, $\mathrm{CoCrFeMnNi}$ and its derivatives have attracted most interest by the scientific community among the class of HEAs so far [26-31]. Its outstanding mechanical properties result from a complex interplay of the impact of lattice distortion, deformation twinning and dislocation slip. The stacking fault energy is strongly depending on the $\mathrm{Fe}$, $\mathrm{Mn}$ and Ni content whereas lattice distortion significantly altered by changing $\mathrm{Cr}$ and $\mathrm{Mn}$ content. In order to experimentally separate these fundamental parameters, HEA samples with deviations from the almost equimolar composition need to be investigated. We hence provide experimental results on chemistry and crystal structure of as-deposited thin films within the Co-Cr-Fe-Mn-Ni system deposited from sectioned targets onto Si substrates by means of magnetron sputtering. The thin films are crystallographically analyzed towards the almost binary border systems. The disorder of the thin film with quinary, (nearly) equimolar composition deposited at the center multi-element target is verified down to atomic length scale and compared to bulk CoCrFeMnNi as well.

\section{Experimental}

Co-Cr-Fe-Mn-Ni thin films were deposited by non-reactive r.f. magnetron sputtering in a Leybold Z 550 PVD coater. For this purpose, a specifically designed segmented sputter target of $152.4 \mathrm{~mm}$ diameter was prepared as shown in Figure 1a. It consists of five segments of same geometry of 
individual pure metal plates that were bound onto a target holder made of copper which provides water cooling and electrical power connection. The individual metallic segments were prepared from $6 \mathrm{~mm}$ thick, $152.4 \mathrm{~mm}$ diameter circular metal plates (Fe, Mn, Cr, Co and Ni by MaTeck GmbH, Germany; each with a purity of $99.98 \%$ and better) by electro discharge machining (EDM). Subsequent to the cutting process, the metal plates were grinded to remove potential contamination resulting from EDM. This segmented sputter target allows for high-throughput preparation of multi-element systems by thin film growth as well as screening of locally resolved phase formation. The vacuum chamber was pumped to a base pressure of $2 \cdot 10^{-4} \mathrm{~Pa}$. Substrates used were $10 \cdot 10 \mathrm{~mm}^{2} \mathrm{Si}$ wafers (Si wafer with 1 $\mu \mathrm{m}$ thermal oxide layer, CrysTec GmbH, Germany); these were placed after ultrasonic bath cleaning in acetone in the vacuum chamber at various defined positions in relation to the segmented sputter target (according to Figure 1b). They correspond to the center position (Pos. 0), an inner circle of small diameter (Pos. 1 to 5) and an outer circle of larger diameter (Pos. 6 to 10) right within the binary systems. Thus, a set of sputtered Co-Cr-Fe-Mn-Ni thin films of various compositions was systematically created in one deposition process, offering the advantage, that all thin films experienced the same plasma conditions and pre-treatment. Prior to the deposition process, the substrates were plasma etched for $5 \mathrm{~min}$ in a $500 \mathrm{~W}$ r.f. plasma of $0.5 \mathrm{~Pa}$ Ar. To enhance adhesion of the Co-Cr-FeMn-Ni films, a $100 \mathrm{~nm}$ thin pure Cr thin film was deposited first; for that, a pure Cr target $(152.4 \mathrm{~mm}$ diameter, 6 mm thick, supply of Cr by FHR Anlagenbau GmbH, Germany) was operated in d.c. mode at $0.5 \mathrm{~Pa} \mathrm{Ar}, 500 \mathrm{~W}$ and $0 \mathrm{~V}$ substrate bias for $20 \mathrm{~s}$. The $\mathrm{Cr}$ and the segmented target were placed in the vacuum chamber opposite to each other, and the sequential depositions of individual layers were done by using an appropriate shutter system. The Co-Cr-Fe-Mn-Ni thin film deposition was carried out at $0.1 \mathrm{~Pa} \mathrm{Ar}, 500 \mathrm{~W}$ and $0 \mathrm{~V}$ substrate bias for $960 \mathrm{~min}$ to achieve thin films of approximately 5 $\mu \mathrm{m}$ in thickness. Bulk CoCrFeMnNi samples in near equilibrium-like condition were prepared from elemental materials mixed in equiatomic concentration by means of arc-melting (AM/0.5 provided by Edmund Bühler $\mathrm{GmbH}$ ). The Ar base pressure for arc-melting was $60 \mathrm{kPa}$ following several alternating iterations of pumping and Ar flooding. Re-melting a $\mathrm{Zr}$ lump in the vacuum chamber was used in order to reduce residual oxygen by liquefying prior to every melting step. The prepared button was flipped and re-melted for at least five times for homogenization. After the final melting step, the 
alloy was cast into a rod-shaped $\mathrm{Cu}$ mold. The diameter and the length of the cast rod were $12 \mathrm{~mm}$ and $60 \mathrm{~mm}$, respectively. For homogenization, the sample was encapsulated into a quartz glass tube under vacuum and annealed at $1200{ }^{\circ} \mathrm{C}$ for $72 \mathrm{~h}$. The chemical composition was analyzed using inductively coupled plasma optical emission spectrometry (ICP-OES). Elemental powders with purities of 99.95 $\%$ and higher were used for validation of the performed X-ray diffraction experiments. system by Bruker equipped with a LynxEye line detector. The Cu tube was operated at $30 \mathrm{kV}$ and 10 $\mathrm{mA}$, and the according radiation was filtered by means of a Ni foil. In order to suppress fluorescence radiation of $\mathrm{Fe}, \mathrm{Co}$ and $\mathrm{Ni}$ a suitable discrimination interval of the LynxEye detector was used.

117 Precise lattice parameters were determined using extrapolation of the peak positions by means of a

118 Nelson-Riley approach [32]. For HCP crystal structure, an adopted least square fitting according to

119 Ref. [33] was used. The digits provided for lattice parameters indicate the error of the measurement

120 which is mainly altered by texture (number of peaks) and peak width. Scanning electron microscopy

121 (SEM) investigations were performed on a Zeiss EVO50 system operated at $25 \mathrm{kV}$ (for complete stimulation of the necessary L-lines of $\mathrm{Co}, \mathrm{Cr}, \mathrm{Fe}, \mathrm{Mn}$ and $\mathrm{Ni}$ for standard-less quantification) equipped with a Thermo Scientific energy-dispersive X-ray spectroscopy (EDX) system. For determination of local chemical composition, five measurements on approx. $200 \cdot 200 \mu \mathrm{m}^{2}$ areas were performed for each wafer position. Characteristic Si lines resulting from the substrate were analyzed for deconvolution of the spectra but omitted during standard-free quantification of the composition. For transmission electron microscopy (TEM), an aberration- corrected (image) FEI Titan 80-300 (FEI, Eindhoven) operated at $300 \mathrm{kV}$ and equipped with field emission gun, Gatan Ultrascan CCD camera (Gatan Inc., Pleasanton, CA), and EDAX S-UTW EDX detector was used. Dark field images (DF) and selected area diffraction (SAD) pattern were acquired to study the local crystallography and microstructure. Scanning-TEM studies were carried out to investigate local concentration changes. Atom probe tomography (APT) was performed in laser mode using a LEAP4000X HR by Cameca. The pulse efficiency (fraction of pulses leading to detection event) was $1 \%$. The pulse rate and energy was $200 \mathrm{kHz}$ and $50 \mathrm{pJ}$, respectively. The tips were cooled down to $50 \mathrm{~K}$. The mass spectra generally contain numerous small peaks besides the expected peaks of $\mathrm{Fe}, \mathrm{Co}, \mathrm{Cr}, \mathrm{Mn}$ and Ni. For both purposes 
TEM and APT, target preparation by in-situ lift-out was performed using a FEI Strata $400 \mathrm{~S}$ (FEI,

Eindhoven) dual electron and focused ion beam (FIB) system (30 kV for ions). Subsequent to Pt deposition for protection of the target, specimen preparation was performed with consecutively decreasing FIB probe currents in order to avoid beam damage. APT specimens were transferred to a pre-sharpened micro-tip coupon (provided by Cameca) and annular milling with decreasing inner radii to achieve tip shaped specimens with an initial tip radius below $100 \mathrm{~nm}$. Finally, a milling step with a closed circle was performed at $5 \mathrm{kV}$ to remove the severely Ga-damaged surface of the tips. Thermodynamic calculations presented in this study were carried out using the thermochemical software FactSage (V6.4) in conjunction with the commercial database FRAN which includes the following elements: Ni, Cr, Mn, Fe, Co, Si, Mo, W, Al, N, O, C.

\section{Results and Discussion}

\section{Chemical composition}

By means of EDX, the chemical composition of thin films was determined at the center of their sample surfaces (the placement of the substrates was according to Figure 1b). The compositions of the thin films are summarized in Table 1. At "Pos. 0" according to Figure 1b, Fe, Mn and Co are equally distributed in the thin film while an enlarged $\mathrm{Ni}$ content (28.0 at\%) and a reduced $\mathrm{Cr}$ content (14.2 at\%) in comparison to the nominal Cantor alloy were observed. For the sake of simplicity, this thin film composition is called almost equimolar composition in the following. Figure 2 visualizes the chemical compositions of the thin films deposited on off-centered substrates (Pos. 1 to 5 and 6 to 10 , respectively). While significant contributions of third elements up to 12.5 at\% (Ni in the case of $\mathrm{CrMn}$ ) are observed in pseudo-binary thin films at the inner circle, these contributions are significantly reduced to values lower than 5 at $\%$ for thin films deposited at the outer rim. In general, contributions of opposite placed elements are in the range of 1 at $\%$ only. An almost equimolar, binary composition is exclusively obtained for CrMn. This indicates a similar sputter yield for these two elements whereas in all other cases deviations from this ideal situation up to 75 at $\%$ of one contributing element were 
observed. Ni exhibits a significantly higher sputter yield in comparison to the other elements which is in good accordance with the enlarged Ni content at the center of the film.

\section{Crystal structure}

164

XRD patterns of the thin films are presented in Figure 3. At the center position (Pos. 0), a FCC solid solution can clearly be revealed for the as-deposited $\mathrm{CoCrFeMnNi}$ thin film. A comparison to bulk $\mathrm{CoCrFeMnNi}$ in Figure 3a reveals significantly broadened reflections in the case of the film indicating microstructural defects or chemical inhomogeneity across the investigated area. Microstructure analyses in the last paragraph of the present article reveal a nanocrystalline, aligned microstructure as a possible origin of the broadened reflections. Moreover, the absence of the $\{220\}$ reflection indicates slight texturing of the film. Evidence for super lattice formation (B2 or L2 ${ }_{1}$ crystal structure) or additional intermetallic phases cannot be found at this stage. The lattice parameter of $0.3597 \mathrm{~nm}$ obtained for bulk CoCrFeMnNi can be reproduced in the thin film exhibiting a value about $0.361 \mathrm{~nm}$ (please see Table 2).

Since chemical compositions of films deposited on substrates which are placed on the inner and outer circle are similar (Table 1), similar crystal structure data was obtained for these films as shown in Figures $3 b$ and $3 c$ as well as in Table 2. For the almost binary Ni-rich Ni-Cr films, a FCC crystal structure is obtained. In equilibrium, up to 50 at $\% \mathrm{Cr}$ can be solved in FCC solid solution at about $1350{ }^{\circ} \mathrm{C}$ [34]. At lower temperatures, $\mathrm{CrNi}_{2}$ occurs. This phase cannot be identified in the films grown at Pos. 1 and 6. Thus, Ni is either supersaturated in the solid solution or remains segregated with a volume fraction below the resolution limit of XRD. Lattice parameters of Ni-rich Ni-Cr FCC solid solutions approach almost $0.36 \mathrm{~nm}$ at maximum solubility [34]. This is in good accordance with the experimentally determined lattice parameter of $0.357 \mathrm{~nm}$ and $0.358 \mathrm{~nm}$ for the films summarized in Table 2 .

The Cr-Mn binary system exhibits a rather complicated phase diagram incorporating numerous intermetallic phases. Nevertheless, the BCC solid solution can solve up to about 70 at $\%$ of $\mathrm{Mn}$ at roughly $1300{ }^{\circ} \mathrm{C}$ [35]. In this case, lattice parameter increases from $0.2885 \mathrm{~nm}$ with 0.00036 $\mathrm{nm} / 10$ at $\%$ [35]. The obtained lattice parameter of $0.289 \mathrm{~nm}$ in the case of the BCC structured thin 
film at Pos. 2 is in good agreement with the according expectation of $0.2894 \mathrm{~nm}$. The thin film at

Pos. 7 was not further processed since partial delamination occurred. This might be attributed to residual stresses resulting from film growth.

The Mn-rich Mn-Fe thin films exhibit the complex cubic (CC) crystal structure of $\alpha-\mathrm{Mn} . \alpha-\mathrm{Mn}$ can solve up to 30 at $\%$ of $\mathrm{Fe}$ in equilibrium condition almost independent of temperature below the $\alpha / \beta$ transition temperature at about $730{ }^{\circ} \mathrm{C}[36]$. In this case, the lattice parameter of the $\mathrm{CC}$ crystal structure decreases with increasing Fe content from $0.89125 \mathrm{~nm}$ to $0.88875 \mathrm{~nm}$ at 30 at\% $\mathrm{Fe}$ [36]. The determined values of 0.8868 (inner circle, Pos. 3, supersaturated at 35.5 at $\%$ ) and $0.8883 \mathrm{~nm}$ (outer circle, Pos. 8, at 20.1 at $\%$ ) agree well with the above mentioned expectation of 0.8869 (extrapolated) and $0.8888 \mathrm{~nm}[36]$, respectively.

Due to the high content of $\mathrm{Co}$ in the case of the Co-Fe thin films, both BCC and $\mathrm{HCP}$ crystal structure are obtained. An obvious texture in the BCC phase does not allow a verification or falsification of the appearance of a B2 super lattice in the case of the cubic phase. Lattice parameters found for the BCC solid solution $(0.285 \mathrm{~nm})$ are in accordance with the expectations from the chemical composition (0.2839 $\mathrm{nm}$ [37]) whereas the HCP crystal structure exhibits strong similarity with pure Co [39] indicating that $\mathrm{Fe}$ is mostly dissolved within the $\mathrm{BCC}$ solid solution.

For the Ni-rich Ni-Co thin films, a FCC crystal structure was found which is expected for equilibrium conditions. For the determined thin film compositions, a lattice parameter of $0.3537 \mathrm{~nm}$ is expected [38] and is in good agreement with the experimentally found values of 0.355 and $0.354 \mathrm{~nm}$, respectively.

\section{Local investigations of the thin film deposited at the center position}

A detailed microstructure investigation of the as-deposited films was done in case of the CoCrFeMnNi thin film at the center position (Pos. 0 in Figure

1b) since it macroscopically compares well to the Cantor alloy. Figure 4a shows a DF image of the thin film microstructure. Columnar grains aligned

212 parallel to the surface normal of the deposited films are clearly visible. The $10 \mu \mathrm{m}$ objective aperture

213 for DF imaging was placed on the $\{400\}$ ring of the FCC structure revealed in Figure $4 \mathrm{~b}$ in the 

$12 \mathrm{~nm}$ crystallite size in the respective direction. Line broadening $\Delta 2 \Theta$ of the diffraction peaks at the angles $\Theta$ as shown in Figure 3a is used for a rough estimate of the crystallite size $D$ in a greater

217 volume of the thin film. The application of the Scherrer equation $D \approx \frac{K \cdot \lambda}{\Delta 2 \Theta \cdot \cos \Theta}[42]$ for the applied

218 wavelength $\lambda$ yields about $10 \mathrm{~nm}$ perpendicular to the growth direction. Hence, a reasonable

219 agreement of XRD and TEM-DF is obtained. Additional defects, namely stacking faults and twin

220 boundaries appearing during film growth, as they are expected for this kind of medium stacking fault 221 energy alloy [43], might contribute to line broadening as well. Slight texturing of the columnar grains 222 with $\langle 100\rangle$ direction parallel to film normal is observed. Potential candidates for secondary phases can be either derived from thermodynamic calculations as shown in Figure 5 or by the decomposition studies performed in Refs. [14-16]. Figure 5 suggests a decomposition of the FCC solid solution into numerous phases at low temperature in equilibrium condition. There are a potential Cr-rich $\sigma$-phase (Ref. [15] and Figure 5), a L1 $1_{0}$-NiMn phase (Ref. [16] and Figure 5, possible chemical congruence with Ref. [14]), a B2-FeCo (Refs. [14,16]) as well as a Cr-rich BCC phase (Ref. [16] and Figure 5). None of these suggested phases were found in the as-deposited thin film. While the identified FCC structure matches perfectly with the macroscopic lattice parameter for the film parallel to the film normal (due to the geometry used in the XRD experiment) presented in Table 2, there is a significant deviation within the film plane. The lattice parameter is increased by approx. $4 \%$ (clearly visible at high angle reflections) indicating residual in-plane tensile stress within the film. Since in the prepared TEM lamella the HEA thin film remains attached to the Cr buffer, thermal oxide layer and parts of the substrate, the residual stress can be taken as representative for this film architecture. In order to reveal if the contrast changes in Figure 4a are attributed to chemical inhomogeneity or diffraction by varying orientations, STEM-EDX line scan was performed and is shown in Figure 6. Despite the high-angle annular dark-field (HAADF) signal is slightly varying, no indications of phase separation on the nanoscale are found. Thus, the DF intensity variations arise from different diffraction conditions for different grain orientations. Further prove of complete disorder and absence of phase separation is

240 provided by means of atom probe tomography and displayed in Figure 7. Both, 3D elemental

241 (Figure 7a) as well as spatial distributions of the elements along a cylinder cut from the reconstructed 
APT tip (Figure 7b) are completely homogeneous. Chemical inhomogeneity on local scale - although present on macroscopic length scale when using the presented deposition technique - does not seem to contribute to the line broadening observed in Figure 3a.

\section{Conclusions}

For the first time, an attempt is presented for the exploration of the phase field of the face centered cubic solid solution in the vicinity of the important equimolar composition of CoCrFeMnNi (Cantor alloy) on the basis of combinatorial thin film deposition from sectioned targets by magnetron sputtering. A variation of the chemical composition from pseudo-binary thin films on substrates placed at the rim of the coated area towards an almost equimolar composition at the center region was achieved. The following conclusions can be drawn:

- Crystal structures of the binary border systems are BCC (Cr-Mn, Fe-Co), FCC (Ni-Cr, Co-Ni, $\mathrm{Ni}-\mathrm{Cr}), \mathrm{HCP}(\mathrm{Fe}-\mathrm{Co})$ and $\mathrm{CC}(\alpha-\mathrm{Mn}$ prototype; $\mathrm{Mn}-\mathrm{Fe})$.

Hence, a suitable platform for future investigations of the entire face centered cubic phase field is provided. The specific arrangement of pure metal plates from the present contribution will allow fast, efficient investigation of subsystems in the vicinity of minimum and maximum mechanical strength on the one hand - for the equimolar subsystems these are $\mathrm{Ni}-\mathrm{Co}$ and $\mathrm{Co}-\mathrm{Cr}-\mathrm{Ni}$, respectively [19]. On the other hand, placing Fe-Mn next to each other and opposite to Co-Ni-Cr provides the possibility to 
access high $\mathrm{Fe}$ and high $\mathrm{Mn}$ alloys which exhibit intense twinning and transformation induced plasticity [41].

The combinatorial approach presented in this contribution might be used as a basis for more advanced investigations by for example using asymmetric target segmentation, variation of deposition parameters for active microstructure determination or heat-treatments of thin films. This can contribute to a more systematic investigation of the composition- microstructure -property correlation and a facilitated development of thermodynamic databases for materials development.

\section{Acknowledgements}

AK thanks the Carl Zeiss Foundation for financial support by a postdoc grant. Financial support by the Deutsche Forschungsgemeinschaft (DFG), grant no. HE 1872/31-1 is gratefully acknowledged. This work was partly carried out with the support of the Karlsruhe Nano Micro Facility (KNMF, www.knmf.kit.edu), a Helmholtz Research Infrastructure at Karlsruhe Institute of Technology (KIT, www.kit.edu). The authors acknowledge the chemical analysis by ICP-OES by T. Bergfeldt. We thank Mr. S. Zils for his strong support in film deposition.

\section{References}

[1] Cantor, B.; Chang, I. T. H.; Knight, P. \& Vincent, A. J. B.: "Microstructural development in equiatomic multicomponent alloys" in Materials Science and Engineering A 375-377 (2004) 213 218

[2] Yeh, J.-W.; Chen, S.-K.; Lin, S.-J.; Gan, J.-Y.; Chin, T.-S.; Shun, T.-T.; Tsau, C.-H. \& Chang, S.Y.: "Nanostructured High-Entropy Alloys with Multiple Principal Elements: Novel Alloy Design Concepts and Outcomes" in Advanced Engineering Materials 6 (2004) 299 - 303

[3] Freudenberger, J; Rafaja, D.; Geissler, D.; Giebeler, L.; Ullrich, C.; Kauffmann, A.; Heilmaier, M. \& Nielsch, K.: "Face centred cubic multi-component equiatomic solid solutions in the Au-Cu-Ni-PdPt system" in Metals 7 (2017) 135 
[4] Senkov, O.N.; Wilks, G.B.; Miracle, D.B.; Chuang, C.P. \& Liaw, P.K.: "Refractory high-entropy alloys" in Intermetallics 18 (2010) 1758 - 1765

[5] Senkov, O.; Scott, J.; Senkova, S.; Miracle, D. \& Woodward, C.: "Microstructure and room temperature properties of a high-entropy TaNbHfZrTi alloy" in Journal of Alloys and Compounds 509 (2011) $6043-6048$

[6] Feuerbacher, M.; Heidelmann, M. \& Thomas, C.: "Hexagonal High-entropy Alloys" in Materials Research Letters 3 (2015) 1 - 6

[7] Yeh, J.-W.; Chen, Y.-L.; Lin, S.-J. \& Chen, S.-K.: "High-Entropy Alloys - A New Era of Exploitation." in Materials Science Forum 560 (2007) 1 - 9

[8] Tsai, M.-H.: "Physical Properties of High Entropy Alloys" in Entropy 15 (2013) 5338-5345

[9] Zhang, Y.; Zuo, T. T.; Tang, Z.; Gao, M. C.; Dahmen, K. A.; Liaw, P. K. \& Lu, Z. P.: "Microstructures and properties of high-entropy alloys." in Progress in Materials Science 61 (2014) 1 $-93$

[10] Tsai, M.-H. \& Yeh, J.-W.: "High-Entropy Alloys: A Critical Review." in Materials Research Letters 2 (2014) 107 - 123

[11] Yeh, J.-W.: "Physical Metallurgy of High-Entropy Alloys." in JOM 67 (2015) 2254-2261

[12] Miracle, D. B.: "Critical Assessment 14: High entropy alloys and their development as structural materials." in Materials Science and Technology 31 (2015) 1142-1147

[13] Pickering, E. J. \& Jones, N. G.: "High-entropy alloys: a critical assessment of their founding principles and future prospects." in International Materials Reviews 61 (2016) 183 - 202

[14] Schuh, B.; Mendez-Martin, F.; Völker, B.; George, E.; Clemens, H.; Pippan, R. \& Hohenwarter, A. : "Mechanical properties, microstructure and thermal stability of a nanocrystalline CoCrFeMnNi high-entropy alloy after severe plastic deformation" in Acta Materialia 96 (2015) 258 - 268 
[15] Pickering, E.; Muñoz-Moreno, R.; Stone, H. \& Jones, N.: "Precipitation in the equiatomic highentropy alloy CrMnFeCoNi" in Scripta Materialia 113 (2016) 106 - 109

[16] Otto, F.; Dlouhý, A.; Pradeep, K.; Kuběnová, M.; Raabe, D.; Eggeler, G. \& George, E.:

"Decomposition of the single-phase high-entropy alloy CrMnFeCoNi after prolonged anneals at intermediate temperatures" in Acta Materialia 112 (2016) 40 - 52

[17] He, J.; Wang, H.; Huang, H.; Xu, X.; Chen, M.; Wu, Y.; Liu, X.; Nieh, T.; An, K. \& Lu, Z.: "A precipitation-hardened high-entropy alloy with outstanding tensile properties" in Acta Materialia 102 (2016) $187-196$

[18] Shun, T.-T.; Hung, C.-H. \& Lee, C.-F. : "The effects of secondary elemental Mo or Ti addition in Al0.3CoCrFeNi high-entropy alloy on age hardening at $700{ }^{\circ} \mathrm{C} "$ in Journal of Alloys and Compounds 495 (2010) $55-58$

[19] Varvenne, C.; Luque, A. \& Curtin, W. A.: "Theory of strengthening in fcc high entropy alloys" in Acta Materialia 118 (2016) 164 - 176

[20] Wilson, P.; Field, R. \& Kaufman, M.: "The use of diffusion multiples to examine the compositional dependence of phase stability and hardness of the Co-Cr-Fe-Mn-Ni high entropy alloy system" in Intermetallics 75 (2016) 15 - 24

[21] Gebhardt, T.; Music, D.; Takahashi, T. \& Schneider, J. M.: "Combinatorial thin film materials science: From alloy discovery and optimization to alloy design" in Thin Solid Films 520 (2012) 5491 5499

[22] Marshal, A.; Pradeep, K.; Music, D.; Zaefferer, S.; De, P. \& Schneider, J.: "Combinatorial synthesis of high entropy alloys: Introduction of a novel, single phase, body-centered-cubic FeMnCoCrAl solid solution" in Journal of Alloys and Compounds 691 (2017) 683 - 689

[23] Otto, F.; Hanold, N. \& George, E.: "Microstructural evolution after thermomechanical processing in an equiatomic, single-phase CoCrFeMnNi high-entropy alloy with special focus on twin boundaries" in Intermetallics 54 (2014) 39 - 48 
[24] Bhattacharjee, P.P.; Sathiaraj, G.D.; Zaid, M.; Gatti, J.R.; Lee, Chi; Tsai, C.-W. und Yeh, J.-W.:

"Microstructure and texture evolution during annealing of equiatomic CoCrFeMnNi high-entropy

[25] Laplanche, G.; Horst, O.; Otto, F.; Eggeler, G. \& George, E.: "Microstructural evolution of a

CoCrFeMnNi high-entropy alloy after swaging and annealing" in Journal of Alloys and Compounds

[26] Gali, A. \& George, E.: "Tensile properties of high- and medium-entropy alloys" in Intermetallics

[27] Wu, Z.; Bei, H.; Pharr, G. \& George, E.: "Temperature dependence of the mechanical properties of equiatomic solid solution alloys with face-centered cubic crystal structures" in Acta Materialia 81

[28] Otto, F.; Dlouhý, A.; Somsen, C.; Bei, H.; Eggeler, G. \& George, E.: "The influences of temperature and microstructure on the tensile properties of a CoCrFeMnNi high-entropy alloy" in Acta Materialia 61 (2013) 5743 - 5755

[29] Gludovatz, B.; Hohenwarter, A.; Catoor, D.; Chang, E. H.; George, E. P. \& Ritchie, R. O.: "A fracture-resistant high-entropy alloy for cryogenic applications" in Science 345 (2014) 1153-1158

Ritchie, R. O.: "Nanoscale origins of the damage tolerance of the high-entropy alloy CrMnFeCoNi" in

Nature Communications (2015) 10143

[31] Laplanche, G.; Kostka, A.; Horst, O.; Eggeler, G. \& George, E.: "Microstructure evolution and critical stress for twinning in the CrMnFeCoNi high-entropy alloy" in Acta Materialia 118 (2016) 152 $-163$

[32] Nelson, J. B. \& Riley, D. P.: "An experimental investigation of extrapolation methods in the 
[33] Tsai, D. S.; Chin, T. S.; Hsu; S. E. \& Hung, M. P.: "A Simple Method for the Determination of

Lattice Parameters from Powder X-ray Diffraction Data" in Materials Transactions JIM 30 (1989) 474

[34] Predel, B.: "Cr-Ni (Chromium-Nickel) " in Landolt-Börnstein - Group IV Physical Chemistry 5D

(Cr-Cs $-C u-Z r)$ by Madelung, O. (ed.) (1994) SpringerMaterials,

http://materials.springer.com/lb/docs/sm lbs 978-3-540-47417-3 995

[35] Predel, B.: "Cr-Mn (Chromium-Manganese)" in Landolt-Börnstein - Group IV Physical

Chemistry 5D (Cr-Cs-Cu-Zr) by Madelung, O. (ed.) (1994) SpringerMaterials,

http://materials.springer.com/lb/docs/sm_lbs_978-3-540-47417-3_989

[36] Predel, B.: "Fe-Mn (Iron-Manganese)" in Landolt-Börnstein - Group IV Physical Chemistry 5E (Dy-Er-Fr-Mo) by Madelung, O. (ed.) (1995) SpringerMaterials, http://materials.springer.com/lb/docs/sm_lbs_978-3-540-48786-9_1315

[37] Predel, B.: "Co-Fe (Cobalt-Iron)" in Landolt-Börnstein - Group IV Physical Chemistry 5C (Ca$C d-C o-Z r)$ by Madelung, O. (ed.) (1993) SpringerMaterials, http://materials.springer.com/lb/docs/sm_lbs_978-3-540-47411-1_911

[38] Predel, B.: "Co-Ni (Cobalt-Nickel)" in Landolt-Börnstein - Group IV Physical Chemistry 5C (Ca$C d-C o-Z r$ ) by Madelung, O. (ed.) (1993) SpringerMaterials, http://materials.springer.com/lb/docs/sm lbs 978-3-540-47411-1 932

[39] Chiarotti, G.: "1.6 Crystal structures and bulk lattice parameters of materials quoted in the volume" in Landolt-Börnstein - Group III Condensed Matter 24A (Structure) by Chiarotti, G. (ed.) (1993) SpringerMaterials, http://materials.springer.com/lb/docs/sm_lbs_978-3-540-47397-8_6

[40] Inorganic Crystal Structure Database: collection code 43058, PDF no. 01-089-2412 32-637

[41] Li, Z.; Pradeep K. G.; Deng, Y.; Raabe, D. \& Tasan, C. C.: "Metastable high-entropy dual-phase alloys overcome the strength-ductility trade-off" in Nature 534 (2016) 227 - 230 
[42] Scherrer, P.: "Bestimmung der Größe und der inneren Struktur von Kolloidteilchen mittels

390 Röntgenstrahlen" in Nachrichten von der Gesellschaft der Wissenschaften zu Göttingen,

391 Mathematisch-Physikalische Klasse 7 (1918) 98-100, German

392 [43] Zaddach, A. J.; Niu, C.; Koch, C. C. \& Irving, D. L.: "Mechanical Properties and Stacking Fault

393 Energies of NiFeCrCoMn High-Entropy Alloy" in JOM 65 (2013) 1780 - 1789

Table 1: Chemical composition of the thin films in at\% (substrates were placed according to the scheme in Figure 1b). Bold numbers indicate major elements.

\begin{tabular}{|c|c|c|c|c|c|c|}
\hline$\#$ & sample & $\mathrm{Cr}$ & Mn & $\mathbf{F e}$ & Co & $\mathbf{N i}$ \\
\hline 0 & $\mathrm{CoCrFeMnNi}$ & 14.2 & 19.4 & 19.0 & 19.4 & 28.0 \\
\hline 1 & $\mathrm{NiCr}$ & 27.8 & 5.4 & 0.7 & 1.2 & 64.9 \\
\hline 2 & $\mathrm{CrMn}$ & 59.3 & 25.8 & 1.4 & 1.0 & 12.5 \\
\hline 3 & $\mathrm{MnFe}$ & 3.5 & 59.4 & 35.5 & 0.8 & 0.9 \\
\hline 4 & FeCo & 1.7 & 7.7 & 17.0 & 67.9 & 5.7 \\
\hline 5 & CoNi & 2.8 & 7.4 & 1.2 & 26.4 & 62.3 \\
\hline 6 & $\mathrm{NiCr}$ & 22.9 & 4.1 & 0.3 & 0.5 & 72.2 \\
\hline 7 & CrMn & 48.5 & 50.2 & 0.7 & 0.1 & 0.5 \\
\hline 8 & $\mathrm{MnFe}$ & 1.6 & 77.2 & 20.1 & 0.8 & 0.3 \\
\hline 9 & FeCo & 1.2 & 4.9 & 17.6 & 74.4 & 1.9 \\
\hline 10 & CoNi & 1.3 & 2.8 & 0.6 & 33.9 & 61.4 \\
\hline- & $\begin{array}{c}\text { CoCrFeMnNi } \\
\text { (bulk sample, } \\
\text { ICP-OES) }\end{array}$ & 19.7 & 19.2 & 20.3 & 20.4 & 20.4 \\
\hline
\end{tabular}


Table 2: Crystallographic data obtained from the XRD patterns presented in Figure 3. In addition, precise lattice parameters from elemental powders are included for comparison. Number of provided digits provide information about the error of the measurement.

\begin{tabular}{|c|c|c|c|c|c|}
\hline \# & sample & condition & prototype & lattice parameter(s) / nm & $\begin{array}{c}\text { reference latt. } \\
\text { parameter(s) / nm }\end{array}$ \\
\hline & $\mathrm{CoCrFeMnNi}$ & $\begin{array}{c}\text { bulk } \\
\text { material }\end{array}$ & $\mathrm{Cu}(\mathrm{FCC})$ & 0.3597 & 0.359 [9] \\
\hline 0 & $\mathrm{CoCrFeMnNi}$ & film & $\mathrm{Cu}(\mathrm{FCC})$ & 0.361 & \\
\hline 1 & $\mathrm{NiCr}$ & film & $\mathrm{Cu}(\mathrm{FCC})$ & 0.358 & $0.3557[34]$ \\
\hline 2 & CrMn & film & $\mathrm{W}(\mathrm{BCC})$ & 0.289 & $0.2894[35]$ \\
\hline 3 & $\mathrm{MnFe}$ & film & $\begin{array}{r}\alpha-\mathrm{Mn} \\
(\mathrm{CC})\end{array}$ & 0.8868 & $0.8869[36]$ \\
\hline 4 & $\mathrm{FeCo}$ & film & $\begin{array}{c}\mathrm{W}(\mathrm{BCC}) \\
\mathrm{Mg} \\
(\mathrm{HCP})\end{array}$ & $\begin{array}{c}0.285 \\
0.251 / 0.409\end{array}$ & $0.2839[37]$ \\
\hline 5 & $\mathrm{CoNi}$ & film & $\mathrm{Cu}(\mathrm{FCC})$ & 0.355 & $0.3537[38]$ \\
\hline 6 & $\mathrm{NiCr}$ & film & $\mathrm{Cu}(\mathrm{FCC})$ & 0.357 & $0.3549[34]$ \\
\hline 7 & $\mathrm{CrMn}$ & film & W (BCC) & $\begin{array}{c}\text { partially } \\
\text { delaminated }\end{array}$ & $0.2903[35]$ \\
\hline 8 & $\mathrm{MnFe}$ & film & $\begin{array}{l}\alpha-\mathrm{Mn} \\
(\mathrm{CC})\end{array}$ & 0.8883 & $0.8888[36]$ \\
\hline 9 & $\mathrm{FeCo}$ & film & $\begin{array}{c}\text { W (BCC) } \\
\mathrm{Mg} \\
(\mathrm{HCP})\end{array}$ & $\begin{array}{c}0.285 \\
0.251 / 0.409\end{array}$ & $0.2839[37]$ \\
\hline \multirow[t]{6}{*}{10} & $\mathrm{CoNi}$ & film & $\mathrm{Cu}(\mathrm{FCC})$ & 0.354 & $0.3 .537[38]$ \\
\hline & Co & powder & $\begin{array}{c}\mathrm{Mg} \\
(\mathrm{HCP})\end{array}$ & $0.25074 / 0.40699$ & $0.25074 / 0.4070$ [39] \\
\hline & $\mathrm{Cr}$ & powder & W (BCC) & 0.28850 & 0.28840 [39] \\
\hline & $\mathrm{Fe}$ & powder & W (BCC) & 0.28665 & 0.286645 [39] \\
\hline & Mn & powder & $\begin{array}{l}\alpha-\mathrm{Mn} \\
(\mathrm{CC})\end{array}$ & 0.89125 & 0.891250 [40] \\
\hline & $\mathrm{Ni}$ & powder & $\mathrm{Cu}(\mathrm{FCC})$ & 0.35241 & 0.35238 [39] \\
\hline
\end{tabular}



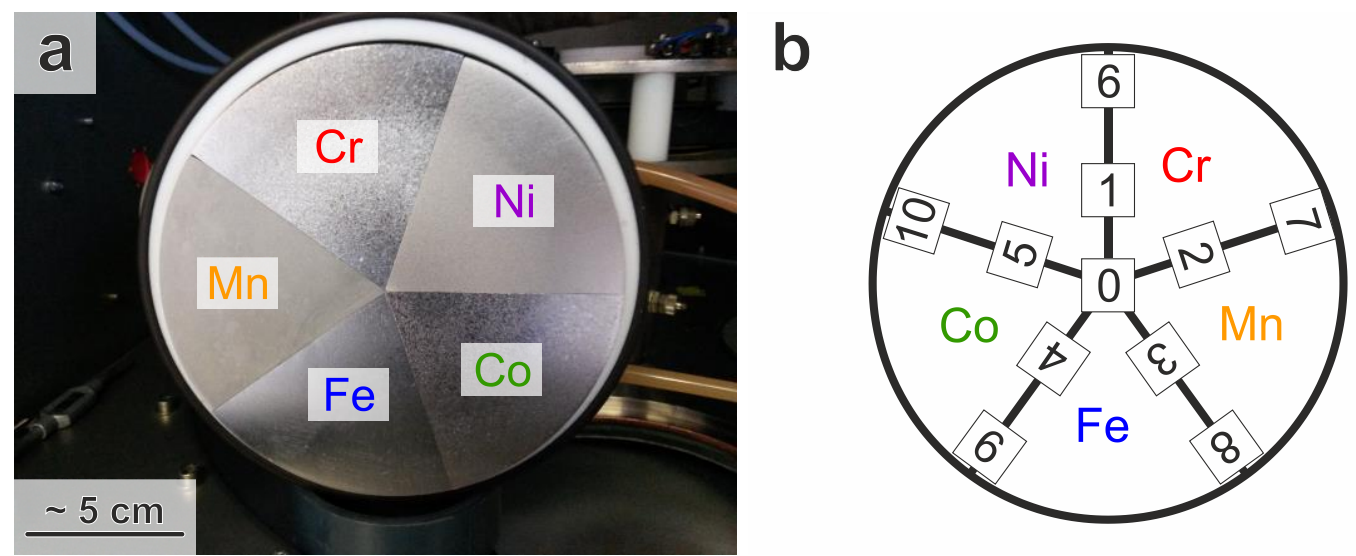

Figure 1: Magnetron sputter target used in this work: a) photograph of mounted target sections and b) scheme of the placement of the eleven Si substrates (no. 0 to 10) underneath the target sections and numbering used throughout this article.
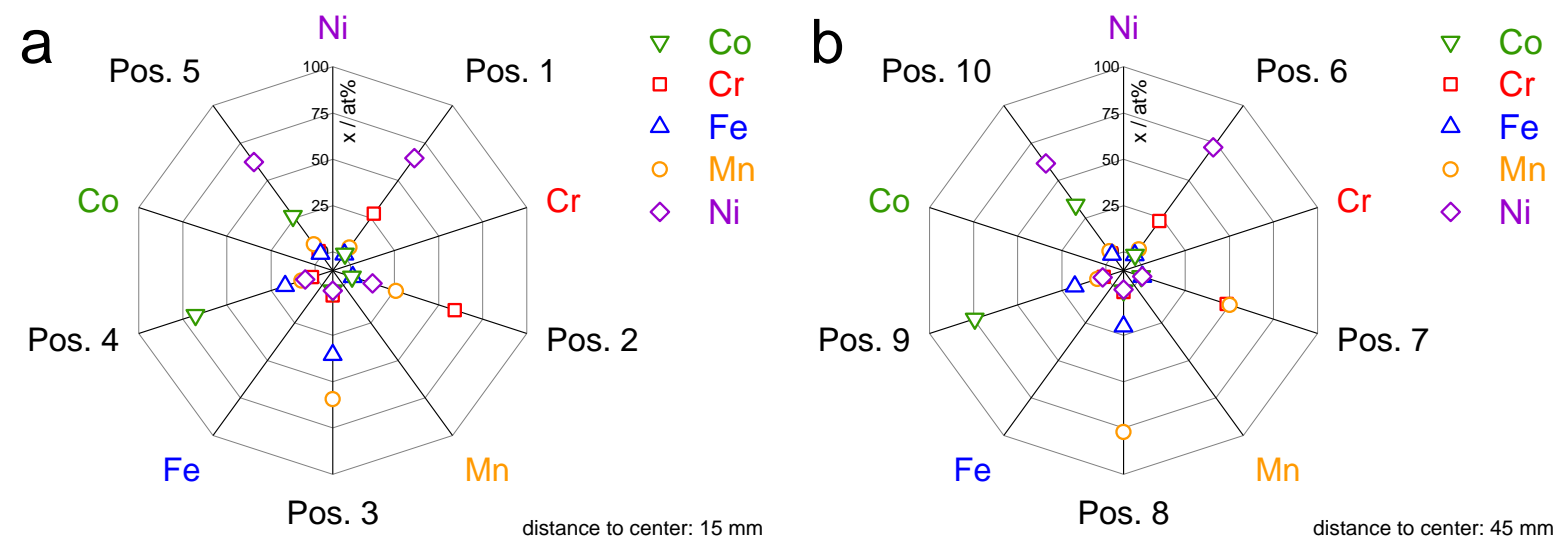

Figure 2: Visualization of chemical compositions of the thin films on substrates placed on the: a) inner, and b) outer circle according to the scheme in Figure $1 \mathrm{~b}$. 

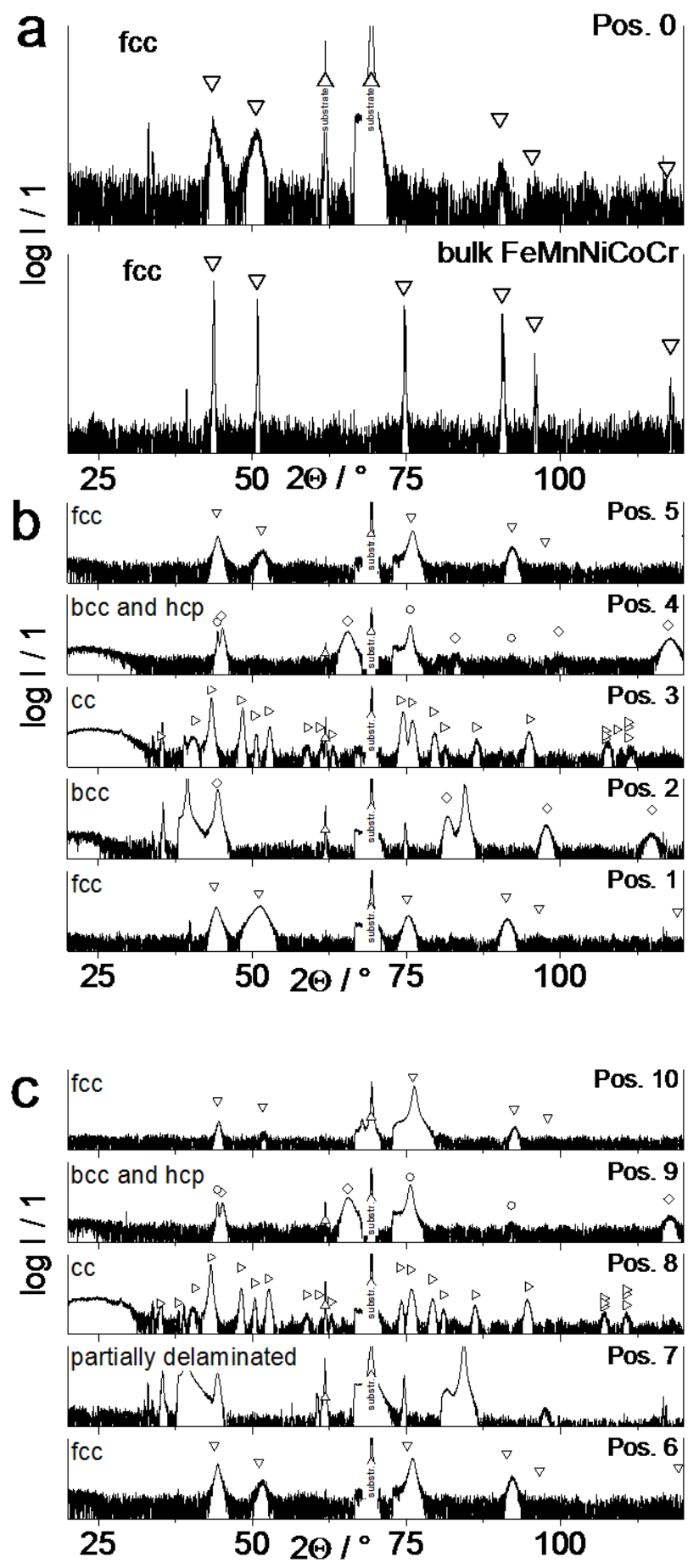

Figure 3: Diffraction patterns (logarithmic intensity scale for the sake of clarity) of: a) CoCrFeMnNi thin film deposited at Pos. 0 in comparison to bulk CoCrFeMnNi, b) thin films on substrates placed on the inner circle (Pos. 1 to 5 ) and c) on the outer circle (Pos. 6 to 10). Phases are indicated by: $\nabla$ Cu prototype (face-centered cubic, fcc), $\diamond \mathrm{W}$ prototype (body-centered cubic, bec), $\bigcirc$ Mg prototype (hexagonally closed packed, hcp), $\triangleright \alpha-M n$ prototype (complex cubic, cc) and $\Delta$ substrate $(\mathrm{Si})$. 


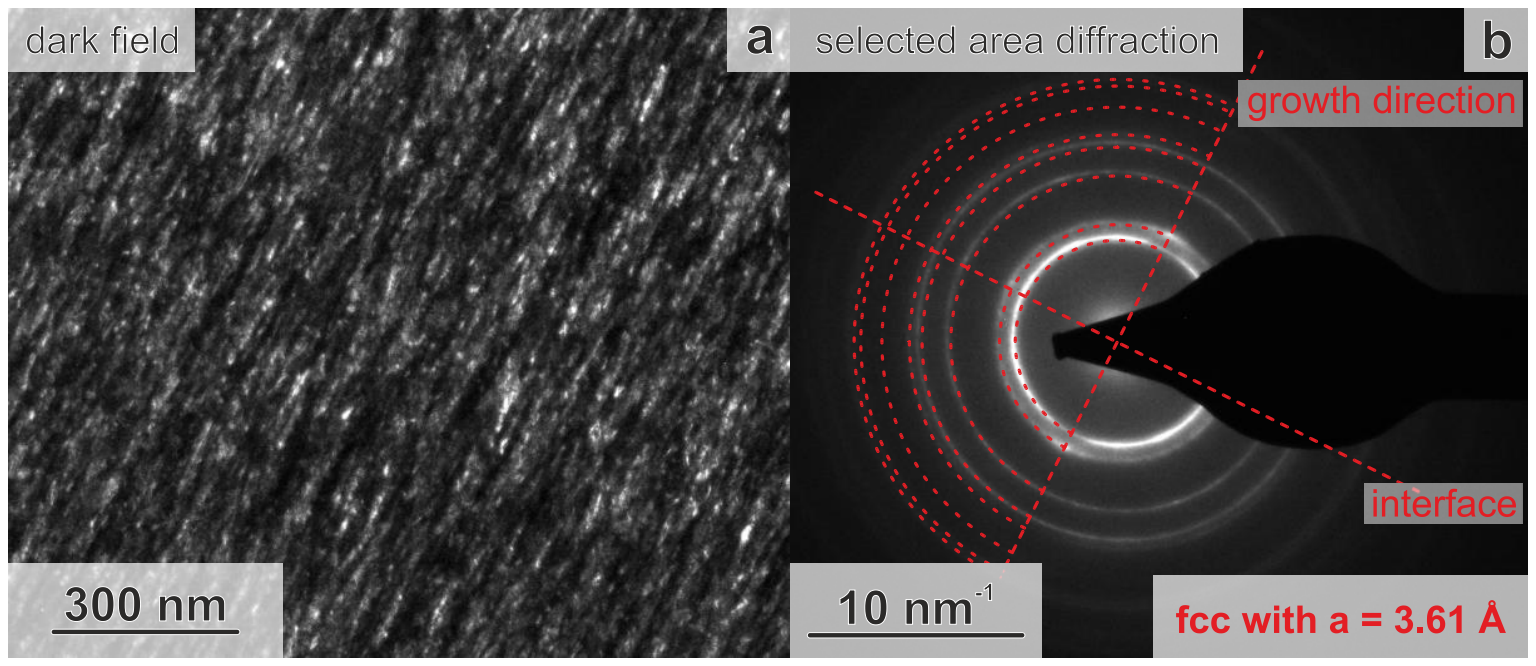

Figure 4: TEM investigations on an in-situ FIB lift-out specimen of the CoCrFeMnNi thin film deposited at Pos. 0 according to Figure 1b: a) DF image using the (400)-ring in approximately the direction of the growth direction and b) SAD pattern with the corresponding FCC rings according to the lattice parameter determined by XRD (please see Table 3).

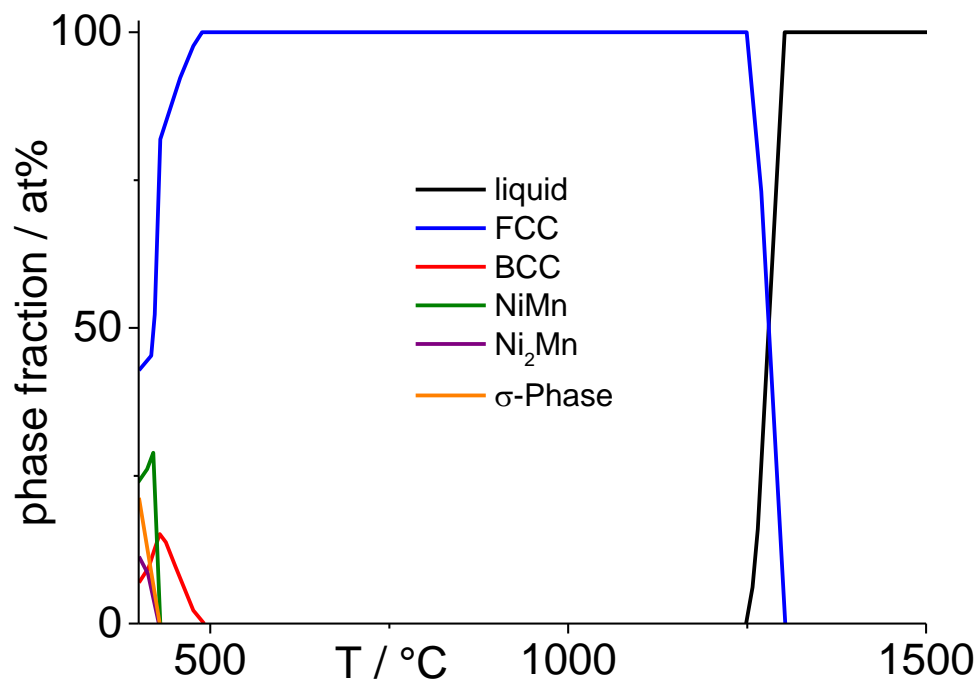

Figure 5: Thermodynamic calculation (utilizing FactSage) of the molar phase fraction as a function of temperature for the composition determined for the CoCrFeMnni thin film deposited at Pos. 0. 


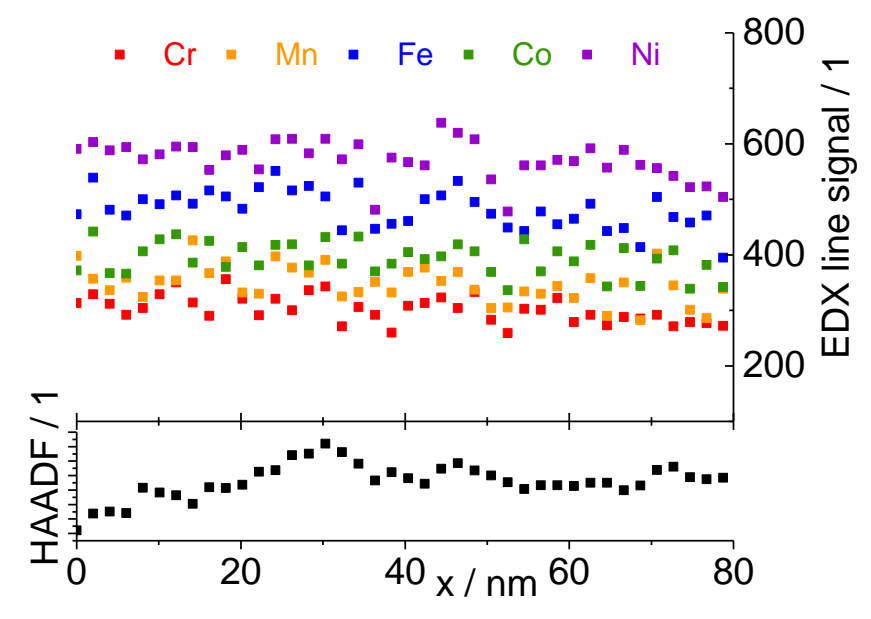

Figure 6: STEM-EDX line scan performed on the in-situ lift-out specimen of the CoCrFeMnNi thin film deposited at Pos. 0. The signal of the EDX lines is uncorrected. The HAADF signal is plotted as reference as well.
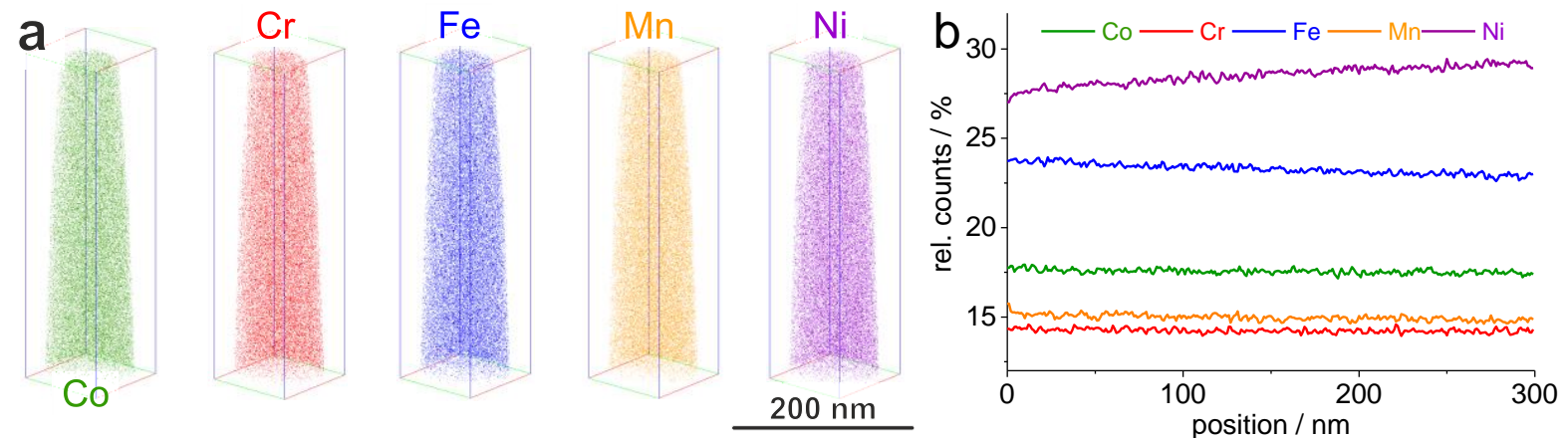

Figure 7: Exemplary APT results on a CoCrFeMnNi thin film specimen taken a film deposited at Pos. 0: a) 3D element distribution and b) spatial element distribution along a cylinder of $\mathbf{5 0} \mathbf{~ n m}$ in diameter (uncorrected rel. counts). The total concentration after deconvolution supports the standard-free EDX including the enlarged Ni content and the $\mathrm{Cr}$ deficite presented in Table 1. 